\title{
Index for EUROSPINE Spring Speciality Meeting, 12-13 May, Krakow, Poland
}

\author{
Abraszko Renata QF2 \\ Andzelina Wolan-Nieroda 3 \\ Bajrovi Fajko F. 8 \\ Balling Horst 17 \\ Berthold Christophe 14 \\ Biaek Micha QF3 \\ Blattert Thomas R. 17 \\ Bommireddy Raj 15 \\ Buchanan Donald 18 \\ Cimerman Matej 8 \\ Clarke Andrew QF7 \\ Dalkilic Ramazan QF6 \\ Daneshi Abdol Hadi 2 \\ Dominguez Dennis E. 1 \\ Dower Ashraf QF2 \\ Efendy Johnny QF2 \\ Ewald Christian QF6 \\ Glinkowski Wojciech 4, 10, 13 \\ Gunness Nitish Vidal 14
}

\author{
Hana Ardian 14 \\ Hara Masahito QF5 \\ Hertel Frank 14 \\ Jablonska-Sudol Katarzyna 3 \\ Jamjoom Bakur 15 \\ Jarmuzek Pawel 7 \\ Jug Marko 8 \\ Kalff Rolf QF6 \\ Kejžar Nataša 8 \\ Klezl Zdenek 15 \\ Latka Dariusz 7 \\ Lee Jun Seok QF1 \\ Maciejczak Andrzej 3 \\ Markmann Yma QF7 \\ Miekisiak Grzegorz 7 \\ Moeri Michaël 1 \\ Mollahousaini Reza 2 \\ Müller Jan-Uwe 5 \\ Nakajima Yasuhiro QF5
}

\author{
Narloch Jerzy 10 \\ Paciak Marek QF3 \\ Pala Adam QF3 \\ Patel Sanjay 15 \\ Pillich Dirk Thomas 5 \\ Racloz Guillaume 1 \\ Rahimizadeh Abolfazl 2 \\ Schrecklinger Wolfram 14 \\ Schroeder Henry Werner Siegfried 5 \\ Sell Philip 9Q, F4 \\ Shimokawa Nobuyuki 16 \\ Shimokawa Nobuyuki 6 \\ Spilsbury Jonathan 18 \\ Szymanski Wojciech 7 \\ Umebayashi Daisuke QF5 \\ Vesel Miloši 8 \\ Yamamoto Yu QF5 \\ Zeden Jan-Philip 5
}

